\title{
Aberrant glycosylation of anti-SARS-CoV-2 IgG is a pro-thrombotic stimulus for platelets
}

Alexander P Bye, ${ }^{1,2}$ Willianne Hoepel, ${ }^{3,4}$ Joanne L Mitchell, ${ }^{1,5}$ Sophie Jégouic, ${ }^{2}$ Silvia Loureiro, ${ }^{2}$ Tanya Sage, ${ }^{1,2}$ Steven de Taeye, ${ }^{6}$ Marit van Gils, ${ }^{6}$ Neline Kriek,,${ }^{1,2}$ Nichola Cooper, ${ }^{7}$ Ian Jones, ${ }^{2}$ Jeroen den Dunnen, ${ }^{3,4}$ Jonathan M Gibbins ${ }^{1,2}$

${ }^{1}$ Institute for Cardiovascular and Metabolic Research, University of Reading, Reading, United Kingdom.

${ }^{2}$ School of Biological Sciences, University of Reading, UK.

${ }^{3}$ Department of Rheumatology and Clinical Immunology, Amsterdam UMC, Amsterdam Rheumatology and Immunology Center, Amsterdam, Netherlands.

${ }^{4}$ Department of Experimental Immunology, Amsterdam UMC, University of Amsterdam, Amsterdam, Netherlands.

${ }^{5}$ Institute of Cardiovascular Sciences, College of Medical and Dental Sciences, University of Birmingham

${ }^{6}$ Department of Medical Microbiology, Amsterdam UMC, University of Amsterdam, Amsterdam Infection and Immunity Institute, Amsterdam, Netherlands

${ }^{7}$ Hammersmith Hospital, Imperial College London, London, UK. 


\section{Abstract}

A subset of patients with COVID-19 become critically ill, suffering from severe respiratory problems and also increased rates of thrombosis. The causes of thrombosis in severely ill COVID-19 patients are still emerging, but the coincidence of critical illness with the timing of the onset of adaptive immunity could implicate an excessive immune response. We hypothesised that platelets might be susceptible to activation by anti-SARS-CoV-2 antibodies and contribute to thrombosis. We found that immune complexes containing recombinant SARS-CoV-2 spike protein and anti-spike IgG enhanced platelet-mediated thrombosis on von Willebrand Factor in vitro, but only when the glycosylation state of the Fc domain was modified to correspond with the aberrant glycosylation previously identified in patients with severe COVID-19. Furthermore, we found that activation was dependent on FcyRIIA and we provide in vitro evidence that this pathogenic platelet activation can be counteracted by therapeutic small molecules R406 (fostamatinib) and ibrutinib that inhibit tyrosine kinases syk and btk respectively or by the P2Y12 antagonist cangrelor.

\section{Introduction}

COVID-19 is more likely to progress to a severe, life threatening condition in patients with pre-existing cardiovascular disease and is associated with dysregulated haemostasis and a high incidence of venous and arterial thromboembolism [1-3]. Emboli in the pulmonary arteries and microthrombi containing fibrin and platelets in the pulmonary microvasculature of COVID-19 patients have been identified at post-mortem [4] and are thought to contribute toward development of acute respiratory distress syndrome (ARDS). It is now believed that multiple factors contribute to the thromboinflammatory state that results in high rates of thrombotic complications. Evidence has indicated the presence of activated vascular endothelial cells, macrophages, platelets, neutrophils and an activated coagulation system in critically ill COVID-19 patients. The mechanistic trigger that causes the changes that accompany an increase in severity in a subset of patients is still the subject of intense research. However, the disparity between the time of peak viral load at 5-6 days after the 
onset of symptoms and occurrence of ARDS after 8-9 days, implicate an excessive immune response rather than direct actions of the virus itself [5].

Further evidence that the adaptive immune response is disturbed in severely ill COVID-19 patients has been provided by a study that found high levels of extrafollicular B cell activation in critically ill patients which correlates with increased morbidity, antibody titres and levels of inflammatory biomarkers [6]. Other studies have also noted the strong association of high antibody titres with disease severity and survival [7, 8]. However, antibodies of severely ill COVID-19 patients have qualitative as well as quantitative differences compared to those with mild illness. Anti-spike IgG in serum samples from severely ill COVID-19 patients were found to have low levels of fucosylatation and elevated galactosylation in the Fc domain $[9,10]$.

Platelets express the antibody receptor FcyRIIA, but it is not known if immune complexes containing afucosylated IgG might activate platelet FcyRIIA. Clustering of platelet FcyRIIA induced by ligand binding triggers intracellular signalling via syk activation and promotes granule secretion and integrin $\alpha_{\| \mathrm{B}} \beta_{3}$ activation [11]. Therefore, activation of FcyRIIA by afucosylated anti SARS-CoV-2 spike lgG might further exacerbate thromboinflammation in critically ill COVID-19 patients.

In this study we investigate the effects of low fucosylation and high galactosylation of antispike $\lg G$ on platelet activation to find the significance of aberrant $\lg G$ glycosylation identified in critically-ill COVID-19 patients on platelet-mediated thrombus formation. We found that potent activation of platelets by immune complexes containing SARS-CoV-2 spike and anti-spike $\lg G$ only occurs when the $\lg G$ expresses both low fucosylation and high galactosylation in the Fc domain and an additional prothrombotic signal, we used vWF, is also present. Enhanced platelet activation and thrombosis, measure in vitro, was sensitive to FcyRIIA inhibition and small molecules inhibitors of syk, btk and P2Y12, suggesting that these therapeutic strategies might reduce platelet-mediated thrombosis in critically-ill COVID-19 patients. 


\section{Materials and Methods}

Spike protein

The sequence of SARS-CoV-2 S1 was obtained from the cloned full-length S sequence and was cloned into the expression vector pTriEx1.1 (EMD Millipore, UK) and characterised as described previously [12]. Sf9 cells were transfected with the baculovirus expression vector FlashBAC Gold (Oxford Expression Technologies, UK) and with the SARS-CoV2-S1 transfer vector to produce recombinant baculovirus. Large-scale protein expression was performed by infecting $1 \mathrm{~L}$ of T.nao38 cells with a high titre stock of the recombinant baculovirus and incubated for $3-5$ days at $27^{\circ} \mathrm{C}$. After incubation the supernatant containing the secreted protein was harvested, clarified by centrifugation at 4,300xg for $20 \mathrm{~min}$ and filtered through a $0.45 \mu \mathrm{m}$ filter. The clear supernatant was supplemented with $0.5 \mathrm{nM}$ nickel sulphate before being loaded onto the Bio-Scale Mini Profinity IMAC Cartridge (Bio-Rad, UK). The elution was carried out at a flow rate of $2.5 \mathrm{ml} / \mathrm{min}$ with a gradient elution of $0.05-0.25 \mathrm{M}$ imidazole over $60 \mathrm{~min}$.

Recombinant anti SARS-CoV-2 IgG

COVA1-18 lgG was produced in 293F cells as described previously [13]. Antibodies with modified glycosylation states were generated and validated as previously described [14]. Validation of the modifications made to COVA1-18 glycosylation are included in table 1.

\section{Blood Preparation}

Blood samples were obtained from healthy donors that had given informed consent and using procedures approved by the University of Reading Research Ethics Committee and collected into vacutainers containing $3.8 \%(\mathrm{w} / \mathrm{v})$ sodium citrate. Platelet-rich plasma (PRP) was prepared by centrifuging whole blood at $100 \mathrm{~g}$ for 20 minutes. Washed platelets were prepared by adding acid citrate dextrose to PRP and centrifuging at $350 \mathrm{~g}$ for 20 minutes and resuspending the platelet pellet at $4 \times 10^{8}$ cells $/ \mathrm{mL}$ in Tyrode's buffer $(\mathrm{NaCl} 134 \mathrm{mM}, \mathrm{KCl}$ 
$2.68 \mathrm{mM}, \mathrm{CaCl}_{2} 1.80 \mathrm{mM}, \mathrm{MgCl}_{2} 1.05 \mathrm{mM}, \mathrm{NaH}_{2} \mathrm{PO}_{4} 417 \mu \mathrm{M}, \mathrm{NaHCO}_{3} 11.9 \mathrm{mM}$, Glucose $5.56 \mathrm{mM}, \mathrm{pH} 7.4)$.

\section{Platelet adhesion assay}

Glass 8 well microslides (Ibidi, Munich, Germany) were coated with $5 \mu \mathrm{g} / \mathrm{ml}$ recombinant SARS-COV-2 spike protein for 60 minutes at $37^{\circ} \mathrm{C}$, washed and then blocked with $10 \%$ fetal calf serum (FCS) for $1 \mathrm{~h}$ at $37^{\circ} \mathrm{C}$. The slides were then washed and treated with $10 \mu \mathrm{g} / \mathrm{ml}$ COVA1-18 recombinant, monoclonal anti-SARS-COV-2 antibodies (see above) for $1 \mathrm{~h}$ at $37^{\circ} \mathrm{C}$. Washed platelets at $2 \times 10^{7}$ cells $/ \mathrm{mL}$ were incubated on the slides for $1 \mathrm{~h}$ at $37^{\circ} \mathrm{C}$. Non-adherent platelets were washed off with Tyrode's buffer and then slides were fixed with $10 \%$ formyl saline for 10 minutes. Wells were then washed and the platelets were labelled with $2 \mu \mathrm{M} \mathrm{DiOC}$. Fluorescence images of adherent platelets were captured with the $20 x$ objective lens of a confocal Ti2 microscope (Nikon).

In vitro thrombus formation

Thrombus formation experiments were performed using microfluidic flow chips (Vena8, CellixLtd, Dublin, Ireland) coated with $5 \mu \mathrm{g} / \mathrm{ml}$ recombinant SARS-COV-2 spike protein for 60 minutes at $37^{\circ} \mathrm{C}$, washed and then blocked with $10 \%$ fetal calf serum (FCS) for $1 \mathrm{~h}$ at $37^{\circ} \mathrm{C}$.

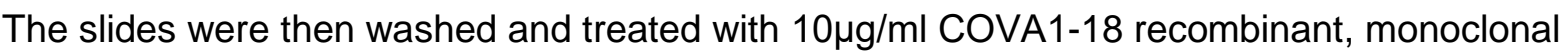
anti-SARS-COV-2 antibodies (see above) for $1 \mathrm{~h}$ at $37^{\circ} \mathrm{C}$ and then $2 \mathrm{U} / \mathrm{ml} \mathrm{vWF}$ for $1 \mathrm{~h}$. Thrombus formation was measured by perfusing citrated whole blood through the flow chambers at $1000 \mathrm{~s}^{-1}$ for 6 minutes before fixing with $10 \%$ formyl saline, staining with $2 \mu \mathrm{M}$ DiOC6 and then imaged by acquiring z-stacks using the $20 \times$ objective lens of a confocal Ti2 fluorescence microscope (Nikon).

\section{Light transmission aggregometry}

Aggregation was measured in washed platelets in half-area 96-well plates (Greiner) by shaking at $1200 \mathrm{rpm}$ for 5 minutes at $37^{\circ} \mathrm{C}$ using a plate shaker (Quantifoil Instruments) after 
stimulating with collagen at a range of concentrations. Changes in light transmittance of 405 nm was measured using a FlexStation 3 plate reader (Molecular Devices).

\section{Flow cytometry measurement of fibrinogen binding}

Measurements of fibrinogen binding were performed using washed platelets pretreated with immune complexes, created by incubating $5 \mu \mathrm{g} / \mathrm{ml}$ recombinant SARS-COV-2 spike protein with $10 \mu \mathrm{g} / \mathrm{ml}$ COVA $1-18$ for 60 minutes at $37^{\circ} \mathrm{C}$, and then stimulated with $1 \mu \mathrm{g} / \mathrm{mL} \mathrm{CRP}$, $10 \mu \mathrm{M}$ ADP or $1 \mu \mathrm{M}$ TRAP- 6 in the presence of fluorescein isothiocyanate-conjugated polyclonal rabbit anti-fibrinogen antibody (Agilent Technologies LDA UK Limited, Cheadle, United Kingdom), and then incubated for 20 minutes in the dark. Platelets were then fixed by addition of filtered formyl saline $(0.2 \%$ formaldehyde in $0.15 \mathrm{M} \mathrm{NaCl})$ and median fluorescence intensities were measured for 5000 platelets per sample on an Accuri C6 Flow Cytometer (BD Biosciences, Berkshire, United Kingdom).

\section{Statistical methods}

Statistical testing as described in figure legends and the results section were performed using GraphPad Prism Software (GraphPad, La Jolla, CA).

\section{Results}

Aberrant glycosylation of anti-spike IgG enhances in vitro thrombus formation on vWF

Previous studies have found a correlation between the severity of COVID-19 and the glycosylation state of anti-SARS-CoV-2 lgG, with low levels of fucosylation and high levels of galactosylation present predominantly in patients with critical illness [9, 10, 14]. To investigate whether anti-SARS-CoV-2 antibodies with aberrant glycosylation activate platelets, and assess the importance of low focusylation and high galactosylation, we studied adhesion and spreading on coverslips coated with IgG-Spike immune complexes containing recombinant SARS-CoV-2 spike protein and COVA1-18 antibody. Four subtypes of COVA118 with different glycosylation states were used in the study; IgG with normal levels of 
fucosylation and galactosylation (WT), a low fucose IgG (Low Fuc), a high galactose IgG (High Gal) and IgG with both low fucose and high galactose (Low Fuc High Gal). We compared the number of platelets adhered to the four different immune complexes relative to spike protein alone and found no significant difference (Figure 1Ai-ii). Levels of platelet adhesion to spike were not significantly greater than adhesion to FCS which was used as a negative control. Adhesive platelet ligands collagen and cross-linked collagen related peptide (CRP-XL) were included as a positive controls. These results indicated that the immune complexes, regardless of the glycosylation state of the anti-spike lgG, were poor ligands for platelet adhesion.

Severe COVID-19 is associated with increased levels of many prothrombotic plasma proteins including von Willebrand Factor (vWF), which has been noted as up to five fold higher (to approx. 5U/ml) in COVID-19 patients in intensive care [15-17]. We combined the immune complex coatings with vWF at $2 \mathrm{U} / \mathrm{ml}$ to simulate a modest increase in plasma vWF levels. The combined immune complex and vWF coating resulted in significantly more platelet adhesion to the Low Fuc High Gal IgG than the WT IgG (Figure 1Bi-ii). Adhesion to $\lg$ G with either Low Fuc or High Gal was not significantly different to the WT IgG. This suggested that both hypofucosylation and hypergalactosylation are required for platelet activation by anti-spike $\lg \mathrm{G}$ immune complexes and that prothrombotic conditions in the circulation of severely ill COVID-19 patients, coupled with dysregulation of IgG glycosylation may result in increased platelet activation.

The increase in platelet adhesion to IgG-spike immune complex and vWF-coated surfaces was modest under static conditions, however, vWF facilitates platelet adhesion to the vascular endothelium in the high shear environment found in arteries and arterioles. To replicate these conditions, we performed thrombus formation experiments in perfusion chambers coated with IgG-spike immune complexes and vWF at a shear rate of $1000 \mathrm{~s}^{-1}$ (Figure 1Ci-ii). Under these conditions there was no significant difference in the average volume of thrombi formed on vWF combined with Low Fuc or High Gal IgG relative to WT 
$\lg$. However, there was a significant increase in thrombus volume formed on vWF in the presence of Low Fuc High Gal lgG immune complexes. This suggests that anti SARS-CoV-2 $\lg \mathrm{G}$ with aberrant glycosylation of the Fc domain synergises with vWF to enhance thrombus formation. This replicates the synergy observed between platelet receptors that predominantly mediate adhesion, such as GPIb and integrin $\alpha 2 \beta 1$, with receptors that strongly activate platelet signalling such as GPVI and CLEC-2 [18].

FcyRIIA is the only Fc receptor expressed in platelets and activates intracellular signalling via Syk activation [19]. We hypothesised FcyRIIA may play the role of a signalling receptor to synergise with the vWF adhesion receptor GPIb to enhance thrombus formation. To test this, whole blood was preincubated with FcyRIIA blocking antibody IV.3 before perfusion through vWF-coated flow chambers with either WT or Low Fuc High Gal immune complexes (Figure 1Di-ii). Blockade of FcyRIIA resulted in a significant reduction in the volume of thrombi formed on the Low Fuc High Gal IgG-containing immune complexes.

Immune complexes presented in suspension do not potently enhance platelet activation

Platelet aggregability is enhanced in patients with severe COVID19 [20] and we hypothesised that this might be due to the presence of immune complexes containing antispike $\lg G$ with aberrant glycosylation of the $\lg G$ Fc domain. To test this hypothesis we preincubated recombinant SARS-COV-2 spike protein with the same four COVA1-18 IgG variants used in previous experiments to enable formation of immune complexes in suspension. We then treated washed human platelets with the immune complexes and stimulated with a range of collagen (type I) concentrations to induce aggregation (Figure 2A). We found that none of the immune complexes enhanced the potency $\left(\mathrm{EC}_{50}\right)$ of collagenevoked aggregation (Figure 2B) or caused aggregation on their own. We also assessed the ability of the immune complexes to potentiate activation of integrin $\alpha_{\| \mathrm{B}} \beta_{3}$ by measuring fibrinogen binding stimulated with ADP, TRAP-6 or CRP-XL by flow cytometry (Figure 2Ciiii). We observed no significant difference between integrin activation stimulated by agonists in the presence of spike protein alone or immune complexes containing both spike and lgG. 
These data suggest that the manner of presentation of immune complexes may be an important part of the mechanism by which they activate platelets. Clustering of FcyRIIA induces intracellular signalling in platelets [19] and it is possible that immune complexes presented in suspension, rather than immobilised on a surface, are below the concentration threshold required to cause clustering of the receptor. Infected endothelial cells may present immobilised immune complexes to platelets in the circulation during severe COVID-19 infection.

Small molecules drugs targeting syk, btk and P2Y12 inhibit lgG-induced potentiation of thrombus formation on vWF in vitro

To understand the signalling processes underpinning the enhancement of thrombus formation on vWF and Low Fuc High Gal lgG and identify potential treatment strategies to counteract pathogenic platelet activation we studied the effects of small molecule inhibitors (Figure 3). FcyRIIA signals through the tyrosine kinase Syk, so we treated whole blood with the Syk inhibitor R406 which is the active metabolite of the FDA-approved drug fostamatinib. Treatment with R406 significantly reduced the volume of thrombi formed on Low Fuc High Gal $\lg G\left(373 k \pm 42 k \mu m^{3}\right)$ relative to vehicle $\left(820 k \pm 172 k \mu m^{3}\right)$, indicating that activation of Syk is important to the prothrombotic effects of aberrantly glycosylated anti-SARs-CoV-2 IgG and that treatment with fostamatinib might be beneficial for patients with severe COVID19 through suppression of IgG-driven platelet activation. FcyRIIA signals via PLCY2 [21] and we hypothesised that, similar to that stimulated by the platelet GPVI receptor [22-24], the pathway involved might be dependent on Btk. We therefore treated platelets with the Btk inhibitor ibrutinib, which is an FDA and EMA approved drug for treatment of B cell cancers. We found that ibrutinib treatment reduced the volume of thrombi formed on the Low Fuc High Gal $\lg G\left(348 \mathrm{k} \pm 68 \mathrm{k} \mu \mathrm{m}^{3}\right)$ to levels similar to the WT IgG $\left(478 \mathrm{k} \pm 76 \mathrm{k} \mu \mathrm{m}^{3}\right)$. Finally, platelet activation via FcyRIIA is dependent on ADP secreted dense granules to activate the P2Y12 receptor and provide the positive feedback signalling required for integrin $\alpha_{\| B} \beta_{3}$ activation and aggregation [25]. Blood samples treated with the P2Y12 antagonist cangrelor, 
were found to reduce the volume of the thrombi formed on Low Fuc High Gal IgG (389k \pm $40 \mathrm{k}^{3}$ ) to comparable levels to those observed with WT IgG.

\section{Discussion}

There is a growing body of evidence that multiple complications arise in severely ill COVID19 patients that increase rates of thrombosis. These include damage to vascular endothelial cells following direct infection with SARS-CoV-2, resulting in disruption of barrier function, exposure of subendothelial collagen as well as release of prothrombotic plasma proteins including vWF from activated endothelial cells [16]. The prothrombotic environment is exacerbated by a cytokine storm that may be driven by activation of macrophages by immune complexes containing afucosylated anti-spike lgG [9]. Hypofucosylated, hypergalactosylated IgG has been identified in the plasma of severely ill COVID-19 patients relative to patients with mild COVID-19 infection and correlated with disease severity $[9,14]$. In the present study we showed that recombinant anti-spike lgG with low levels of fucosylation and high levels of galactosylation activate platelets to enhance thrombus formation on vWF, which is also elevated in severely ill COVID-19 patients [15-17].

We hypothesise that immune complexes present in the pulmonary circulation of severely ill patients might activate platelets and contribute to high rates of thrombosis (Figure 4). A similar mechanism has been identified in severe H1N1 infection, whereby immune complexes present in the lungs activate platelets via FcyRIIA [26]. The role of aberrant IgG glycosylation in stimulating this response was not investigated, but it has been suggested that afucosylated IgG may be common to immune responses against all enveloped viruses [9].

The role of platelets in COVID-19 is still emerging but platelet rich thrombi have been identified in both large arteries and microthrombi [4]. Platelets contain many inflammatory mediators within granules that might contribute toward the flood of cytokines present in critically ill COVID-19 patients. There is still scant information regarding the efficacy of 
antiplatelet drugs in COVID-19 patients but one study has suggested that patients receiving antiplatelet therapy with aspirin prior to hospital admission for COVID-19 appear to be partially protected and have better outcomes, while a separate study found that in-hospital treatment of patients with aspirin reduced mortality $[27,28]$. Other non-antiplatelet drugs in trials for COVID-19 therapy have well documented antiplatelet effects. The Bruton's tyrosine kinase (Btk) inhibitor acalabrutinib has been evaluated in clinical trials on the basis of its potential to block macrophage activation [29], however, the potential of Btk inhibitors to reduce the contribution of platelets to thrombosis in COVID-19 infection have also been noted [30]. We found that the btk inhibitor ibrutinib reversed the enhancement of thrombus formation on vWF caused by IgG with low fucosylation and high galactosylation, supporting the hypothesis that this strategy might have dual benefits on macrophage and platelet activation. The syk inhibitor fostamatinib was identified as a potential COVID-19 therapeutic in a high content screen of drugs that might protect against acute lung injury [31] . The active metabolite of fostamatinib, R406 inhibits release of neutrophil extracellular NETS [32] and macrophage activation induced by plasma from COVID-19 patients [14]. R406 is also known to have potent antiplatelet effects downstream of the GPVI and CLEC-2 receptors [33] and is currently in clinical trials for COVID-19 therapy in the US (NCT04579393) and UK (NCT04581954). We found that R406 reversed the potentiation of thrombus formation on vWF. This suggests that potential COVID-19 therapies such as fostamatinib or acalabrutinib, targeting syk or btk respectively, may be effective not only in limiting the inflammatory response, but also in reducing platelet-mediated thrombosis.

\section{Acknowledgements}

This work was supported by Imperial College NIHR bioresources and grants from the British Heart Foundation (RG/15/2/31224 and RG/20/7/34866), ZonMw (10430 012010008 ) and an Amsterdam Infection \& Immunity COVID-19 grant (24184). 


\section{References}

1. Klok, F.A., et al., Incidence of thrombotic complications in critically ill ICU patients with COVID-19. Thromb Res, 2020. 191: p. 145-147.

2. Tang, N., et al., Abnormal coagulation parameters are associated with poor prognosis in patients with novel coronavirus pneumonia. J Thromb Haemost, 2020. 18(4): p. 844-847.

3. Middeldorp, S., et al., Incidence of venous thromboembolism in hospitalized patients with COVID-19. J Thromb Haemost, 2020. 18(8): p. 1995-2002.

4. $\quad$ Rapkiewicz, A.V., et al., Megakaryocytes and platelet-fibrin thrombi characterize multi-organ thrombosis at autopsy in COVID-19: A case series. EClinicalMedicine, 2020. 24: p. 100434.

5. Tay, M.Z., et al., The trinity of COVID-19: immunity, inflammation and intervention. Nat Rev Immunol, 2020. 20(6): p. 363-374.

6. Woodruff, M.C., et al., Extrafollicular B cell responses correlate with neutralizing antibodies and morbidity in COVID-19. Nat Immunol, 2020. 21(12): p. 1506-1516.

7. Chen, Y., et al., Quick COVID-19 Healers Sustain Anti-SARS-CoV-2 Antibody Production. Cell, 2020. 183(6): p. 1496-1507 e16.

8. Garcia-Beltran, W.F., et al., COVID-19-neutralizing antibodies predict disease severity and survival. Cell, 2021. 184(2): p. 476-488 e11.

9. Larsen, M.D., et al., Afucosylated IgG characterizes enveloped viral responses and correlates with COVID-19 severity. Science, 2020.

10. Chakraborty, S., et al., Proinflammatory IgG Fc structures in patients with severe COVID-19. Nat Immunol, 2021. 22(1): p. 67-73.

11. Arman, M. and K. Krauel, Human platelet IgG Fc receptor FcgammaRIIA in immunity and thrombosis. J Thromb Haemost, 2015. 13(6): p. 893-908.

12. Jegouic, S.M., et al., Recombinant SARS-CoV-2 spike proteins for sero-surveillance and epitope mapping. bioRxiv, 2020.

13. Brouwer, P.J.M., et al., Potent neutralizing antibodies from COVID-19 patients define multiple targets of vulnerability. Science, 2020. 369(6504): p. 643-650.

14. Hoepel, W., et al., Anti-SARS-CoV-2 Ig G from severely ill COVID-19 patients promotes macrophage hyper-inflammatory responses. bioRxiv, 2020.

15. Panigada, M., et al., Hypercoagulability of COVID-19 patients in intensive care unit: A report of thromboelastography findings and other parameters of hemostasis. J Thromb Haemost, 2020. 18(7): p. 1738-1742.

16. Goshua, G., et al., Endotheliopathy in COVID-19-associated coagulopathy: evidence from a single-centre, cross-sectional study. Lancet Haematol, 2020. 7(8): p. e575-e582.

17. Escher, R., N. Breakey, and B. Lammle, Severe COVID-19 infection associated with endothelial activation. Thromb Res, 2020. 190: p. 62.

18. de Witt, S.M., et al., Identification of platelet function defects by multi-parameter assessment of thrombus formation. Nat Commun, 2014. 5: p. 4257.

19. Chacko, G.W., et al., Clustering of the platelet Fc gamma receptor induces noncovalent association with the tyrosine kinase p72syk. J Biol Chem, 1994. 269(51): p. 32435-40.

20. Manne, B.K., et al., Platelet gene expression and function in patients with COVID-19. Blood, 2020. 136(11): p. 1317-1329.

21. Boylan, B., et al., Identification of FcgammaRIla as the ITAM-bearing receptor mediating alphallbbeta3 outside-in integrin signaling in human platelets. Blood, 2008. 112(7): p. 27806.

22. Nicolson, P.L.R., et al., Inhibition of Btk by Btk-specific concentrations of ibrutinib and acalabrutinib delays but does not block platelet aggregation mediated by glycoprotein VI. Haematologica, 2018. 103(12): p. 2097-2108.

23. Quek, L.S., J. Bolen, and S.P. Watson, A role for Bruton's tyrosine kinase (Btk) in platelet activation by collagen. Curr Biol, 1998. 8(20): p. 1137-40. 
24. Bye, A.P., et al., Severe platelet dysfunction in NHL patients receiving ibrutinib is absent in patients receiving acalabrutinib. Blood Adv, 2017. 1(26): p. 2610-2623.

25. Stolla, M., et al., CaIDAG-GEFI deficiency protects mice in a novel model of Fcgamma RIIAmediated thrombosis and thrombocytopenia. Blood, 2011. 118(4): p. 1113-20.

26. Boilard, E., et al., Influenza virus H1N1 activates platelets through FcgammaRIIA signaling and thrombin generation. Blood, 2014. 123(18): p. 2854-63.

27. Chow, J.H., et al., Aspirin Use is Associated with Decreased Mechanical Ventilation, ICU Admission, and In-Hospital Mortality in Hospitalized Patients with COVID-19. Anesth Analg, 2020.

28. Meizlish, M.L., et al., Intermediate-dose anticoagulation, aspirin, and in-hospital mortality in COVID-19: a propensity score-matched analysis. Am J Hematol, 2021.

29. Roschewski, M., et al., Inhibition of Bruton tyrosine kinase in patients with severe COVID-19. Sci Immunol, 2020. 5(48).

30. Nicolson, P.L., et al., A rationale for blocking thromboinflammation in COVID-19 with Btk inhibitors. Platelets, 2020. 31(5): p. 685-690.

31. Kost-Alimova, M., et al., A High-Content Screen for Mucin-1-Reducing Compounds Identifies Fostamatinib as a Candidate for Rapid Repurposing for Acute Lung Injury. Cell Rep Med, 2020. 1(8): p. 100137.

32. Strich, J.R., et al., Fostamatinib Inhibits Neutrophils Extracellular Traps Induced by COVID-19 Patient Plasma: A Potential Therapeutic. J Infect Dis, 2020.

33. Spalton, J.C., et al., The novel Syk inhibitor R406 reveals mechanistic differences in the initiation of GPVI and CLEC-2 signaling in platelets. J Thromb Haemost, 2009. 7(7): p. 1192-9. 


\section{Figure 1. Low fucosylation and high galactosylation of the IgG $F$ tail enhances} adhesion to von Willebrand Factor. Platelet adhesion to slides coated with immune complexes containing recombinant SARS-CoV-2 spike protein and COVA1-18 recombinant anti-spike lgG with modified glyocosylation. (Ai) Numbers of platelets adhered to glass slides coated with FCS (negative control), spike protein only or spike protein plus unmodified lgG (WT) or IgG modified to have low fucosylation (low fuc), high galactosylation (high gal) or both (low fuc high gal) and (Aii) representative images of adhered platelets stained with DiOC6. (Bi) Numbers of platelets adhered to vWF plus immune complexes containing spike protein and modified lgGs and (Bii) representative images of adhered platelets. (Ci) Volume of thrombi formed on vWF with immune complexes containing spike and either WT IgG or IgG with modified glyosylation and (Cii) representative images of thrombi stained with DiOC6. (Di) Volume of thrombi formed on vWF plus WT IgG or IgG with low fucosylation and high galactosylation in the presence or absence of $20 \mu \mathrm{g} / \mathrm{ml} \mathrm{IV.3} \mathrm{and} \mathrm{(Dii)} \mathrm{representative}$ images of thrombi stained with DiOC6. Values are mean \pm s.e.m. Significant differences were tested by 2 -way ANOVA with the Tukey multiple comparisons test, ${ }^{*} p<0.05,{ }^{* *} p<$ 0.01 .

\section{Figure 2. Aggregation and integrin $\alpha_{11 B} \beta_{3}$ activation are unaffected by COVID19} immune complexes. (Ai) Concentration response curves plotting platelet aggregation following stimulation with a range of type I collagen concentrations (from $10 \mu \mathrm{g} / \mathrm{ml}$ to $10 \mathrm{ng} / \mathrm{ml}$ ) in the presence of immune complexes containing spike plus WT $\lg$ G or $\lg G$ with modified glycosylation and $(\mathrm{Bi})$ scatter plots of $\log \mathrm{EC}_{50}$ for collagen in the presence of the different treatments. Fibrinogen binding to platelets measured by flow cytometry following stimulation with (Ci) $10 \mu \mathrm{M}$ ADP, (Cii) $1 \mu \mathrm{g} / \mathrm{ml} \mathrm{CRP}-\mathrm{XL}$, (Ciii) $1 \mu \mathrm{M}$ TRAP-6 in the presence of spike only or immune complexes containing WT IgG or IgG with modified gycosylation. Significant differences were tested by 2-way ANOVA with the Tukey multiple comparisons test.

\section{Figure 3. Prothrombotic activity of low fucose, high galactose IgG1 immune} complexes is inhibited by Syk, Btk or P2Y12 inhibition. (A) Volume of thrombi formed in perfusion chambers on vWF plus immune complexes containing spike protein plus either WT $\lg G$ or IgG modified to have low fucosylation and high galactosylation following treatment with vehicle (DMSO), $1 \mu \mathrm{M}$ R406, $1 \mu \mathrm{m}$ ibrutinib or $1 \mu \mathrm{M}$ cangrelor and (B) representative images of thrombi stained with DiOC6. Values are mean \pm s.e.m. Significant differences were tested by 2 -way ANOVA with the Tukey multiple comparisons test, ${ }^{*} p<0.05,{ }^{* *} p<$ 0.01 . 
Figure 4. Aberrant glycosylation of anti-spike IgG in immune complexes act in concert with VWF to enhance platelet thrombus formation. SARS-CoV-2 infects vascular endothelial cells, and combined with other inflammatory signals, results in endothelial activation and release of pro-thrombotic factors including VWF. After the onset of adaptive immunity, anti-spike $\lg G$ accumulates in the circulation and binds to SARS-CoV-2. In critically ill COVID-19 patients, anti-spike IgG has abnormally low levels of fucosylation and high levels of galactosylation. Immune complexes containing this aberrant glycosylation pattern activate platelet FcyRIIA which stimulate intracellular signals that synergise with the adhesive ligand vWF to promote platelet activation and thrombus formation. Schematic was created with BioRender.com.

\begin{tabular}{l|c|c|c|c|} 
& $\begin{array}{c}\text { Fucosylation } \\
(\%)\end{array}$ & $\begin{array}{c}\text { Galactosylation } \\
(\%)\end{array}$ & $\begin{array}{c}\text { Sialylation } \\
(\%)\end{array}$ & $\begin{array}{c}\text { Bisection } \\
(\%)\end{array}$ \\
\hline COVA1-18 & 97.8 & 19.6 & 1.1 & 2.4 \\
COVA1-18 low fucose & 8.7 & 17.4 & 0.7 & 0.3 \\
COVA1-18 high galactose & 98.1 & 83.0 & 11.3 & 1.0 \\
COVA1-18 low fucose high galactose & 9.1 & 77.6 & 5.4 & 0.2
\end{tabular}

Table 1. Glycosylation of WT and modified COVA1-18 IgG. 


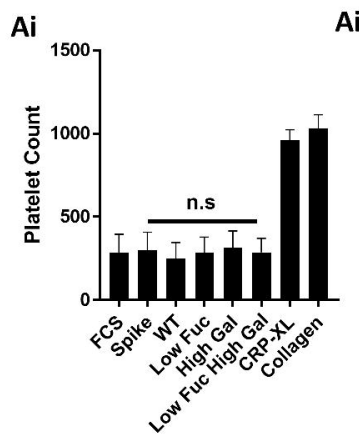

$\mathrm{Ci}$

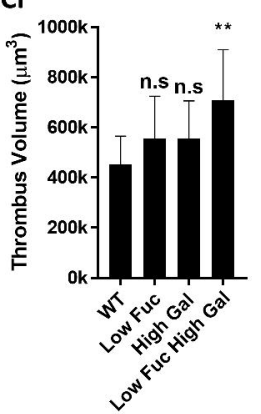

Aii

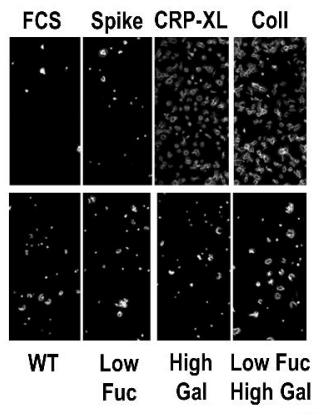

Cii

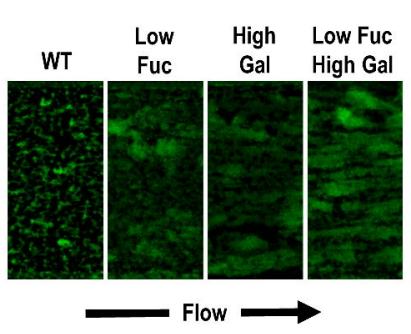

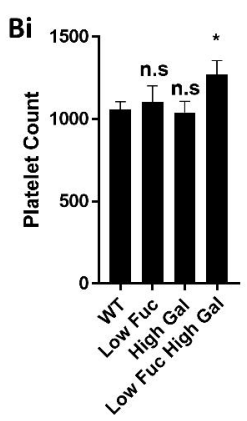

Di

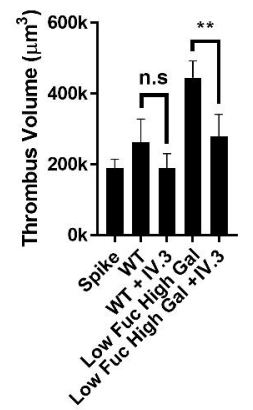

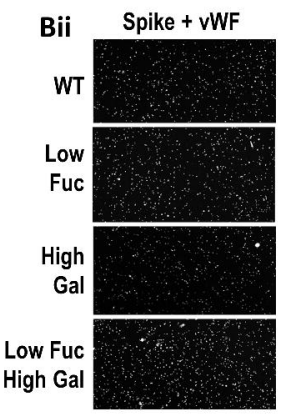

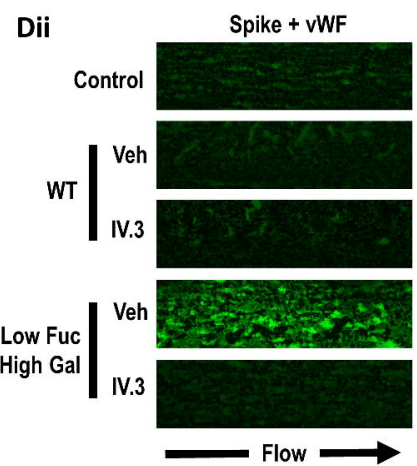



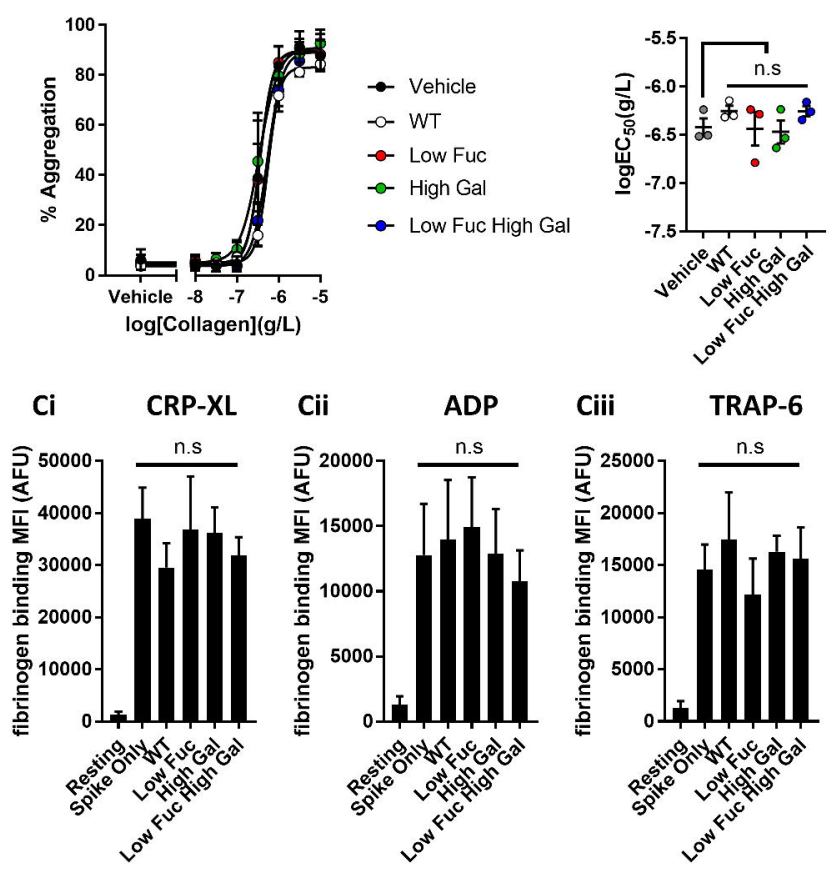
A

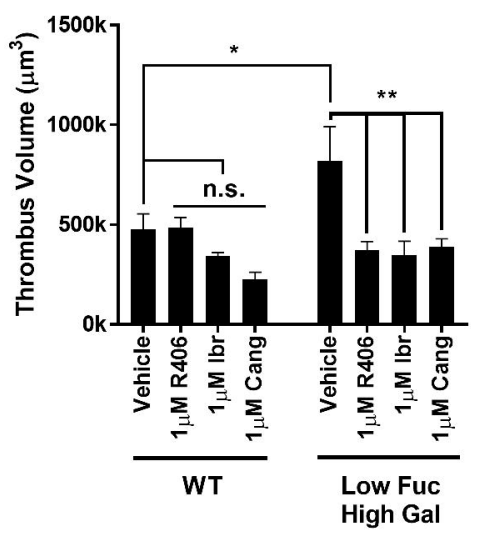

B

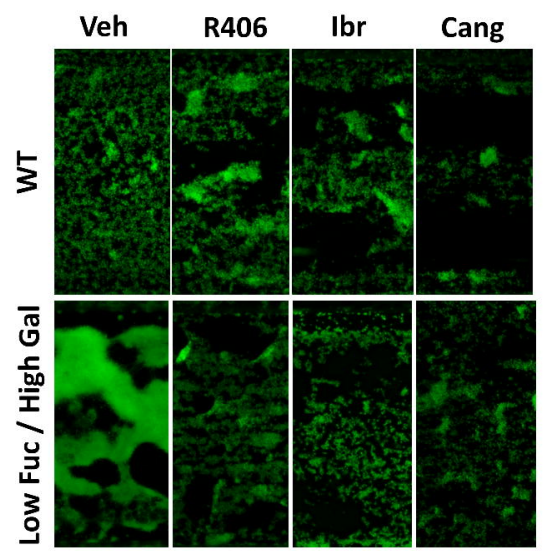




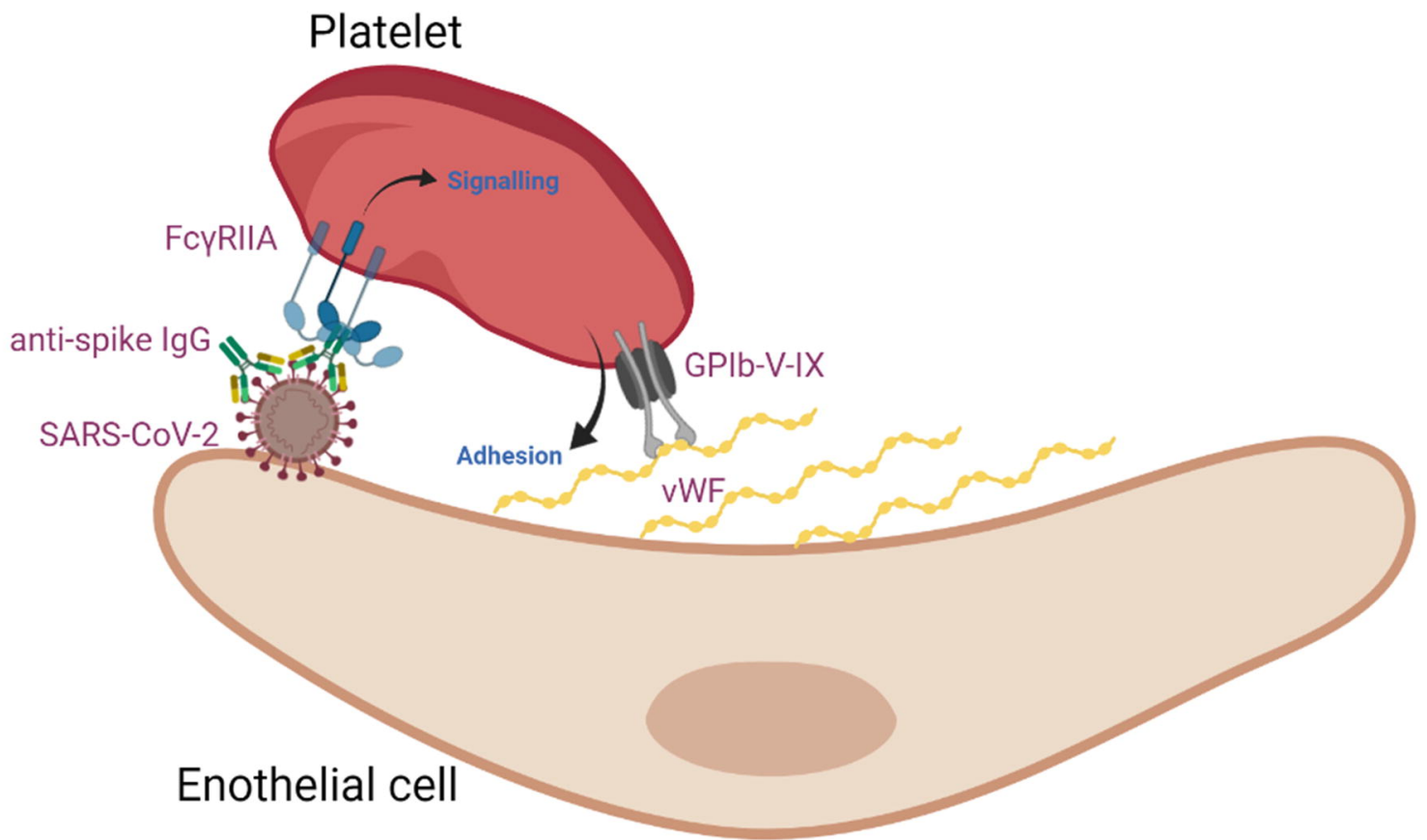

